\title{
Profile of oritavancin and its potential in the treatment of acute bacterial skin structure infections
}

\section{Subhashis Mitra \\ Usman Saeed \\ Daniel H Havlichek \\ Gary E Stein}

Department of Infectious Diseases, Michigan State University,

East Lansing, MI, USA
Correspondence: Gary E Stein Department of Infectious Diseases, Michigan State University, 1355 Bogue Street, B 323 Life Sciences Building, East Lansing, MI 48824, USA Email gary.stein@hc.msu.edu
This article was published in the following Dove Press journal:

Infection and Drug Resistance

6 July 2015

Number of times this article has been viewed

\begin{abstract}
Oritavancin, a semisynthetic derivative of the glycopeptide antibiotic chloroeremomycin, received the US Food and Drug Administration approval for the treatment of acute bacterial skin and skin structure infections caused by susceptible Gram-positive bacteria in adults in August 2014. This novel second-generation semisynthetic lipoglycopeptide antibiotic has activity against a broad spectrum of Gram-positive bacteria, including methicillin-resistant Staphylococcus aureus (MRSA), vancomycin-intermediate S. aureus (VISA), and vancomycin-resistant Enterococcus. Oritavancin inhibits bacterial cell wall synthesis and is rapidly bactericidal against many Gram-positive pathogens. The long half-life of this drug enables a single-dose administration. Oritavancin is not metabolized in the body, and the unchanged drug is slowly excreted by the kidneys. In two large Phase III randomized, double-blind, clinical trials, oritavancin was found to be non-inferior to vancomycin in achieving the primary composite end point in the treatment of acute Gram-positive skin and skin structure infections. Adverse effects noted were mostly mild with nausea, headache, and vomiting being the most common reported side effects. Oritavancin has emerged as another useful antimicrobial agent for treatment of acute Gram-positive skin and skin structure infections, including those caused by MRSA and VISA.
\end{abstract}

Keywords: antibiotic, Gram-positive bacteria, MRSA, VRSA, vancomycin, MIC

\section{Introduction}

The emergence of antibiotic resistance among Gram-positive organisms has been increasing over several decades. ${ }^{1}$ Unfortunately, the antibiotic pipeline has not kept pace with the increasing demand for combating resistant pathogens. Resistance among Staphylococcus aureus and Enterococcus spp. particularly to vancomycin has emerged as a major concern. The need for expanding the antibiotic armamentarium for treatment of complicated infections including acute bacterial skin and skin structure infections (ABSSSIs) is desperately increasing. In this regard, the recent US Food and Drug Administration (FDA) approval of a few antibiotics for treatment of ABSSSI is welcome news. Oritavancin, a semisynthetic lipoglycopeptide antibiotic, was approved by the FDA for the treatment of acute Gram-positive skin and skin structure infections in August 2014. ${ }^{2}$

This article reviews our current knowledge of oritavancin including the clinical trials that demonstrate the efficacy and safety of the drug for ABSSSIs.

\section{Chemistry}

Oritavancin is a second-generation lipoglycopeptide (has both hydrophobic and lipophilic groups) and contains a heptapeptide core similar to other members of the glycopeptide group. ${ }^{3}$ A synthetic derivative of the naturally occurring glycopeptide 
chloroeremomycin, oritavancin, differs from its parent compound by addition of a $4^{\prime}$-chlorobiphenylmethyl substituent on the disaccharide sugar. The chemical structure of oritavancin also differs from vancomycin by virtue of an additional monosaccharide moiety (4-epi-vancosamine) attached to the amino acid residue on ring 6 (Figure 1). These structural alterations confer significantly enhanced activity against vancomycin-resistant Enterococci and vancomycinintermediate and vancomycin-resistant Staphylococcus. Oritavancin can also bind to D-alanyl-D-lactate in addition to D-alanyl-D-alanyl (D-Ala-D-Ala), and the affinity is enhanced by its ability to form dimers prior to attachment to bacterial peptidoglycan cell wall. This dimerization is possible due to interactions between the disaccharides attached to residue 4 , the chlorine on ring 2 , and the 4-epi-vancosamine on ring $6 .^{4-6}$

\section{Mechanism of action}

The antibacterial activity of oritavancin occurs by multiple mechanisms, resulting in concentration-dependent killing of susceptible Gram-positive pathogens. The drug not only exhibits rapid bactericidal effect on exponentially growing cells but has also been shown to be active against stationaryphase cells of susceptible Gram-positive bacteria including vancomycin-resistant $S$. aureus (VRSA). ${ }^{7}$ Furthermore, oritavancin also demonstrated activity against $S$. aureus biofilms in vitro. ${ }^{7}$ Oritavancin may also have some effect on inhibition of RNA synthesis. ${ }^{6,8}$

Like other members of the glycopeptide class, oritavancin inhibits bacterial cell wall synthesis by blocking the transglycosylation step during peptidoglycan synthesis. The stoichiometric complex produced by binding of

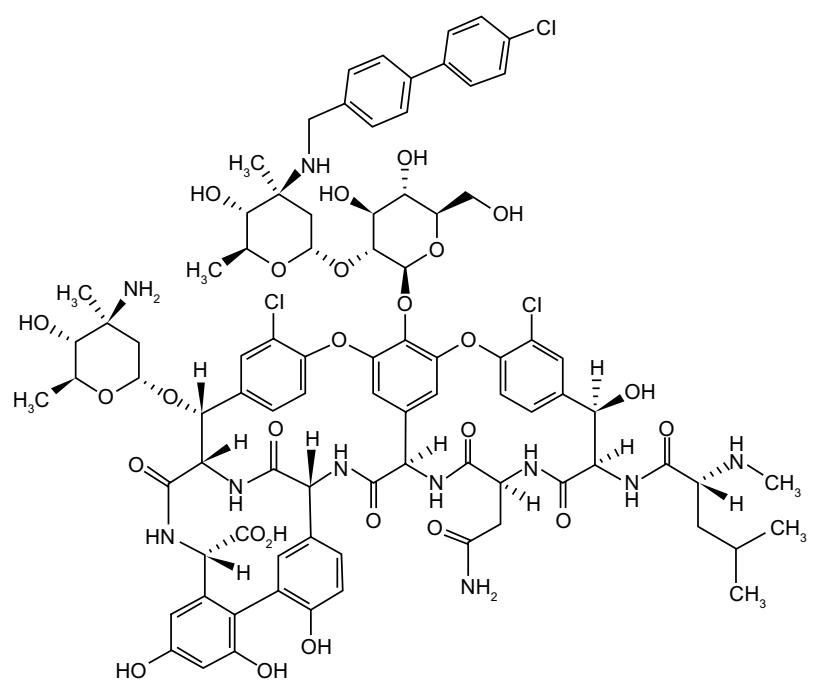

Figure I Chemical structure of oritavancin. oritavancin to carboxyl terminal acyl-D-alanyl-D-alanine residues of the pentapeptide moiety of lipid II prevents incorporation of the disaccharide-pentapeptide monomer into nascent peptidoglycan by the transglycosylase enzyme. ${ }^{4}$ The hydrophobic 4 '-chlorobiphenylmethyl side chain on the disaccharide sugar also facilitates attachment to bacterial cell membrane, providing additional stability during oritavancin's interaction with lipid II. ${ }^{6}$ The binding of the drug to pentapeptides of the peptidoglycan is further enhanced by formation of dimers as mentioned previously.

Another important mechanism of action, that has been reported, is the ability of oritavancin to inhibit transpeptidation. This is attributed to the $4^{\prime}$-chlorobiphenylmethyl side chain, which allows binding of the drug to a secondary site in peptidoglycan, the pentaglycyl bridge, which likely contributes to oritavancin's activity against vancomycin-intermediate S. aureus (VISA) and vancomycin-resistant Enterococcus isolates.

The 4'-chlorobiphenylmethyl side chain of the molecule is also credited for Gram-positive bacterial cell death by membrane depolarization and increased membrane permeability. ${ }^{9}$ This mechanism, which leads to disruption of membrane ultrastructure, has been demonstrated in vitro to support both antibacterial activity against stationary-phase inocula of staphylococci and rapid killing of susceptible Gram-positive pathogens. $^{7,9}$

Oritavancin can accumulate within eukaryotic cells, reaching macrophage intracellular concentrations 200 times above extracellular concentrations after 24 hours of in vitro incubation. ${ }^{10}$ The accumulation of the drug had no effect on the bactericidal activity of macrophages. These findings suggest that oritavancin possesses high efficacy against intracellular staphylococci and provides reassurance that oritavancin accumulation does not inhibit phagocytic killing of macrophages. ${ }^{10}$

\section{Antimicrobial activity and resistance}

Oritavancin has demonstrated excellent in vitro activity against Gram-positive aerobic and anaerobic bacteria, including staphylococci, streptococci, enterococci, and Clostridium difficile. ${ }^{11}$ Table 1 summarizes the available data on the susceptibility of Gram-positive organisms to oritavancin, while Table 2 compares other parenteral agents that might be considered for the treatment of Gram-positive infections in different geographic regions. ${ }^{12,13}$

Oritavancin's minimum inhibitory concentration (MIC) required to inhibit the growth of $90 \%$ of organisms (MIC90) for methicillin-resistant $S$. aureus (MRSA) was $0.06 \mu \mathrm{g} / \mathrm{mL}$, 
Table I Susceptibility of aerobic and anaerobic Gram-positive bacteria to oritavancin

\begin{tabular}{|c|c|c|c|c|}
\hline Organism & Number of isolates & MIC range $(\mu \mathrm{g} / \mathrm{mL})$ & MIC50 $(\mu \mathrm{g} / \mathrm{mL})$ & MIC90 $(\mu \mathrm{g} / \mathrm{mL})$ \\
\hline \multicolumn{5}{|c|}{ Susceptibility of aerobic Gram-positive bacteria to oritavancin ${ }^{12,13}$} \\
\hline Methicillin-susceptible Staphylococcus aureus & 7,332 & $\leq 0.004-0.50$ & 0.03 & 0.06 \\
\hline Methicillin-resistant Staphylococcus aureus & 4,264 & $\leq 0.004-0.25$ & 0.03 & 0.06 \\
\hline Staphylococcus epidermidis & 586 & $\leq 0.008-0.5$ & $0.03-0.06$ & $0.06-0.12$ \\
\hline Enterococcus faecalis & 1,017 & $\leq 0.004-0.5$ & $0.015-0.03$ & 0.06 \\
\hline Vancomycin-susceptible Enterococcus faecium & 278 & $\leq 0.004-0.03$ & $\leq 0.008$ & $\leq 0.008-0.015$ \\
\hline Vancomycin-resistant Enterococcus faecium & 216 & $\leq 0.004-0.5$ & $0.008-0.06$ & $0.06-0.12$ \\
\hline Streptococcus anginosus group & 172 & $\leq 0.008-0.06$ & $\leq 0.008$ & $\leq 0.008-0.015$ \\
\hline Streptococcus pyogenes & 868 & $\leq 0.0005-1.0$ & 0.03 & $0.03-0.25$ \\
\hline Streptococcus agalactiae & 546 & $0.00 \mathrm{I}-0.5$ & 0.03 & 0.12 \\
\hline Streptococcus dysgalactiae & 81 & $\leq 0.008-0.5$ & 0.06 & $0.25-0.5$ \\
\hline \multicolumn{5}{|c|}{ Susceptibility of anaerobic Gram-positive bacteria to oritavancin" } \\
\hline Clostridium perfringens & 28 & $0.25-1$ & 0.5 & 1.0 \\
\hline Propionibacterium acnes & 11 & $0.125-0.25$ & 0.125 & 0.25 \\
\hline Peptostreptococcus anaerobius & 15 & $0.06-0.5$ & 0.125 & 0.25 \\
\hline Peptoniphilus asaccharolyticus & 17 & $\leq 0.03-0.5$ & 0.25 & 0.5 \\
\hline Anaerococcus prevotti & 16 & $\leq 0.03-1$ & $\leq 0.03$ & 0.25 \\
\hline Micromonas micros & 15 & $\leq 0.03-0.5$ & 0.125 & 0.25 \\
\hline
\end{tabular}

Abbreviations: MIC, minimum inhibitory concentration; MIC50, MIC required to inhibit the growth of $50 \%$ of isolate; MIC 90 , MIC required to inhibit the growth of $90 \%$ of isolate.

while the MIC required to inhibit the growth of $50 \%$ of organisms (MIC50) was $0.03 \mu \mathrm{g} / \mathrm{mL}$. Oritavancin's MIC against MRSA isolates from the USA and Europe was at least eight times lower compared to other antimicrobial agents (Table 2). ${ }^{12}$ It was also observed that oritavancin MIC appears to mirror vancomycin MIC. The modal oritavancin MIC increased with increasing vancomycin MIC in both geographic regions. ${ }^{12}$

Table 2 Susceptibility of Gram-positive bacteria to oritavancin and selected antibiotics in different geographic regions

\begin{tabular}{|c|c|c|c|c|}
\hline \multirow[t]{2}{*}{ Organism } & \multirow[t]{2}{*}{ Antimicrobial agent } & \multicolumn{3}{|c|}{$\operatorname{MIC90}(\mu \mathrm{g} / \mathrm{mL})$} \\
\hline & & USA & Europe & Canada \\
\hline \multirow[t]{3}{*}{ Methicillin-resistant Staphylococcus aureus } & Oritavancin & 0.06 & 0.06 & 0.06 \\
\hline & Vancomycin & I & 1 & I \\
\hline & Daptomycin & 0.5 & 0.5 & 0.5 \\
\hline \multirow[t]{3}{*}{ Coagulase-negative staphylococci } & Oritavancin & 0.06 & 0.06 & 0.12 (Staphylococcus epidermidis) \\
\hline & Vancomycin & 2 & 2 & 2 (S. epidermidis) \\
\hline & Daptomycin & 0.5 & 0.5 & 0.25 (S. epidermidis) \\
\hline \multirow[t]{3}{*}{ Enterococcus faecalis } & Oritavancin & 0.06 & 0.06 & 0.06 \\
\hline & Vancomycin & 2 & 2 & 2 \\
\hline & Daptomycin & 2 & 1 & 2 \\
\hline \multirow[t]{3}{*}{ Vancomycin-susceptible Enterococcus faecium } & Oritavancin & $\leq 0.008$ & $\leq 0.008$ & 0.015 \\
\hline & Vancomycin & 2 & 1 & 1 \\
\hline & Daptomycin & 4 & 4 & 2 \\
\hline \multirow[t]{3}{*}{ Vancomycin-resistant Enterococcus faecium } & Oritavancin & 0.12 & 0.06 & 0.12 \\
\hline & Vancomycin & $>16$ & $>16$ & $>32$ \\
\hline & Daptomycin & 2 & 4 & 2 \\
\hline \multirow[t]{3}{*}{ Streptococcus agalactiae } & Oritavancin & 0.12 & 0.12 & 0.12 \\
\hline & Vancomycin & 0.5 & 0.5 & 0.5 \\
\hline & Daptomycin & 0.25 & 0.25 & 0.25 \\
\hline \multirow[t]{3}{*}{ Streptococcus pyogenes } & Oritavancin & 0.12 & 0.12 & 0.25 \\
\hline & Vancomycin & 0.5 & 0.5 & 0.5 \\
\hline & Daptomycin & $\leq 0.06$ & $\leq 0.06$ & 0.12 \\
\hline \multirow[t]{3}{*}{ Streptococcus anginosus group } & Oritavancin & 0.015 & $\leq 0.008$ & NR \\
\hline & Vancomycin & 1 & 1 & NR \\
\hline & Daptomycin & 0.5 & 0.5 & NR \\
\hline
\end{tabular}

Notes: Data from Mendes et al' ${ }^{12}$ and Karlowsky et al. ${ }^{13}$

Abbreviations: MIC90, minimum inhibitory concentration required to inhibit the growth of $90 \%$ of isolate; NR, not reported. 
An interesting observation, related to the binding of oritavancin to plastic surfaces, questioned the accuracy of oritavancin susceptibility assays performed by the broth microdilution technique without using the surfactant polysorbate $80 .{ }^{14}$ The omission of polysorbate 80 in those experiments likely resulted in loss of the drug to plastic surfaces and may have underestimated the potency of oritavancin. In subsequent studies, the addition of polysorbate 80 at a concentration of $0.002 \%$ throughout all steps of the broth microdilution assay resulted in several-fold reductions in oritavancin MICs against S. aureus and Enterococcus faecalis. ${ }^{14}$ The use of polysorbate 80 is now the standard for testing in vitro oritavancin MICs. ${ }^{12}$

Oritavancin demonstrated synergistic and bactericidal effect against VISA and VRSA isolates in combination with gentamicin or linezolid, while synergy with rifampin against VRSA isolate was bacteriostatic. ${ }^{8}$

Resistance to oritavancin among clinical isolates has not been detected so far. ${ }^{6,15}$ However, moderate level of resistance (MIC $\leq 16 \mu \mathrm{g} / \mathrm{mL}$ ) to the drug has been observed in the laboratory among Enterococcus isolates demonstrating the VanA and $\operatorname{VanB}$ phenotypes and can occur by various mechanisms. ${ }^{16}$ Total replacement of the peptidoglycan precursors terminating in D-alanine by isolates capable of producing peptidoglycan precursors terminating in D-lactate can confer resistance to oritavancin. This can be achieved in vitro by either increasing resistance gene expression or reducing production of D-Ala-D-Ala. Resistance to the drug may also occur with expression of the $v a n Z$ gene. Additionally, mutations in the $v a n S_{B}$ sensor gene of the $v a n B$ cluster confer cross-resistance to teicoplanin and oritavancin. ${ }^{16}$ Thus, it is quite likely that emergence of resistance to oritavancin may occur with widespread clinical use.

\section{Pharmacokinetics/ pharmacodynamics}

Oritavancin is available as a sterile white-to-off-white lyophilized powder, and each $50 \mathrm{~mL}$ capacity glass vial contains $400 \mathrm{mg}$ of oritavancin base, mannitol, and phosphoric acid (The Medicines Company, Parsippany, NJ, USA). The drug is poorly absorbed across an intact gastrointestinal tract due to its high molecular weight and needs to be administered intravenously, after each vial is reconstituted with sterile water for injection and subsequently diluted with $5 \%$ dextrose in sterile water. The use of normal saline for dilution may cause precipitation of the drug. ${ }^{3}$

Pharmacokinetic parameters of oritavancin are summarized in Table $3 .{ }^{17}$ After an intravenous (IV) dose, oritavancin
Table 3 Pharmacokinetic parameters of oritavancin in Phase II/II studies $(n=360)$

\begin{tabular}{ll}
\hline Pharmacokinetic parameter & Mean value (SD) \\
\hline$C_{\max }(\mu \mathrm{g} / \mathrm{mL})$ & $28.5(12.2)$ \\
$C_{\min }(\mu \mathrm{g} / \mathrm{mL})$ & $1.99(1.10)$ \\
$A \cup C_{0-24}(\mu \mathrm{g} \mathrm{h} / \mathrm{mL})$ & $146(63.7)$ \\
$T_{1 / 2} \beta$ (hours) & $31.2(11.4)$ \\
$T_{1 / 2} \gamma$ (hours) & $393(73.5)$ \\
$\mathrm{CL}(\mathrm{L} / \mathrm{h})$ & $0.601(0.204)$ \\
$V_{c}(\mathrm{~L})$ & $7.10(2.46)$
\end{tabular}

Notes: Data from Rubino et al. ${ }^{17}$

Abbreviations: SD, standard deviation; $C_{\max }$ maximum plasma concentration; $C_{\min }$, minimum plasma concentration; $A \cup C_{0-24}$ area under the plasma concentration-time curve from time 0 hours to 24 hours; $T_{1 / 2} \beta$, plasma elimination-phase half-life; $T_{1 / 2} \gamma$, plasma terminal elimination half-life; $C L$, total clearance; $V_{c}$, volume of distribution of the central compartment.

achieves a plasma concentration that is best illustrated using a three-compartment model. The initial rapid distribution phase is followed by a slightly slower and longer second elimination phase of 12-24 hours. The final phase has a very long terminal half-life of 393 hours in humans due to slow release of the drug from tissue accumulation sites. The FDA-approved dose of 1,200 mg given as a single IV dose over 3 hours for treatment of ABSSSIs is predicted to start at terminal half-life concentration of 5-20 $\mu \mathrm{g} / \mathrm{mL} .{ }^{18}$ However, the free-drug concentration is expected to be significantly less as $85 \%-90 \%$ of oritavancin is protein bound. The drug displayed linear kinetics when administered as single or multiple IV doses ranging from $100 \mathrm{mg}$ to $800 \mathrm{mg}$ or weight-based doses ranging from $0.02 \mathrm{mg} / \mathrm{kg}$ to $10 \mathrm{mg} / \mathrm{kg}$ of body weight. ${ }^{19}$

Oritavancin has extensive tissue distribution with a volume of distribution of about $1 \mathrm{~L} / \mathrm{kg}$ and accumulates in macrophages, reaching intracellular concentrations 200 times above extracellular concentrations after 24 hours of in vitro incubation. ${ }^{10}$ Using a cantharide-induced blister fluid model, the mean blister fluid/plasma ratio was calculated to be 0.19 (19\%), and the mean drug concentrations in blister fluid exceeded the oritavancin MIC90 for S. aureus by approximately 2-5.5 times..$^{20}$ The maximal concentration of the drug was achieved in blister fluid about 10 hours after dosing and was undetectable at 100-150 hours after the last dose. ${ }^{20}$

Oritavancin demonstrates concentration-dependent killing against susceptible Gram-positive bacteria. The long plasma half-life of oritavancin makes it difficult to conclusively identify the best pharmacodynamic parameter, though the observation that the lowest colony count was observed with a single large dose of the drug suggests that the ratio between the maximum serum concentrations and MIC of the organism $\left(C_{\max } / \mathrm{MIC}\right)$ best predicts the drug's bactericidal efficacy. ${ }^{21}$ However, other pharmacodynamic 
parameters, the time during which the concentration of drug in plasma exceeds the MIC ( $T>$ MIC) and area under the concentration-time curve to MIC ratio (AUC/MIC), may also correlate with efficacy. ${ }^{21}$

Oritavancin is not metabolized, and metabolites have not been detected in either urine or feces. The drug is slowly eliminated from tissue sites with approximately $6 \%$ of the dose eliminated from the body over 7 days after a singledose IV infusion, mostly excreted unchanged in urine with less than $1 \%$ eliminated in the feces.

\section{Animal models}

The efficacy of oritavancin against drug-resistant bacterial pathogens has been demonstrated in various animal models. A single dose of the drug was able to clear bacteremia in a rat central venous catheter (CVC) model of infection caused by a strain of Enterococcus faecium with the VanA phenotype. ${ }^{22}$ None of the animals in the oritavancin group showed evidence of metastatic disease compared to $100 \%$ of the controls. The organism was recovered from the explanted CVCs in only $12.5 \%$, compared to $87.5 \%$ of untreated animals. ${ }^{22}$ Oritavancin was found to be as effective as vancomycin in clearing bacteremia and reducing bacterial counts in vegetations and tissues in a rabbit model of experimental left-sided MRSA endocarditis. ${ }^{23}$ The drug was found to be active against VanA and $\operatorname{Van} B$ strains of $E$. faecalis in a rabbit aortic endocarditis model, while vancomycin did not show activity against the VanB-type strain, and teicoplanin was not active against the VanA-type strain. ${ }^{24}$ The addition of gentamicin in this endocarditis model enhanced the bactericidal activity of oritavancin against vancomycin-resistant $E$. faecalis and prevented the emergence of resistant strains. ${ }^{25}$

The efficacy of oritavancin against Streptococcus pneumoniae was evaluated in a neutropenic mouse pneumonia model. ${ }^{18}$ Pulmonary infection in mice resulted from aspiration or inhalation of the inoculum from the nasopharynx. Single IV oritavancin dose of $\geq 10 \mathrm{mg} / \mathrm{kg}$ was active against S. pneumoniae, achieving $\geq 3 \operatorname{logs}$ of killing at 72 hours. The addition of 5\% surfactant resulted in eight times increase in MIC against $S$. pneumoniae to $0.008 \mu \mathrm{g} / \mathrm{mL}$ and 16 times increase in MIC against $S$. aureus to $1 \mu \mathrm{g} / \mathrm{mL}$, despite the use of $0.002 \%$ polysorbate 80 . This implies that some binding of oritavancin to surfactant occurs, though this is significantly less than the 256 times increase in MIC against $S$. aureus observed for daptomycin after addition of surfactant. ${ }^{18}$

The activity of oritavancin was studied in a rabbit model of pneumococcal meningitis. ${ }^{26}$ Cerebrospinal fluid (CSF) penetration of the drug was estimated to be
$1 \%-5 \%$ of the serum concentration, and the maximum CSF concentration was achieved several hours after a single IV dose. Overall, a single IV dose of oritavancin was slightly less efficacious compared to a 12-hour continuous infusion of ceftriaxone, though this was statistically not significant. However, the culture samples obtained from oritavancintreated animals were sterile after 1 hour. ${ }^{26}$ In a similar rabbit model of cephalosporin-resistant pneumococcal meningitis, oritavancin was found to be highly effective either alone or in combination with ceftriaxone, though no synergistic effect was observed. ${ }^{27} \mathrm{~A}$ decrease in CSF inflammatory markers was noted with the combination of oritavancin, ceftriaxone, and dexamethasone achieving the greatest magnitude reduction. Two therapeutic failures were observed with the oritavancin and dexamethasone combination, though the combination was found to be rapidly bactericidal. CSF oritavancin level was lower in the groups treated with dexamethasone, though this was statistically not significant. ${ }^{27}$

Oritavancin has been shown to prevent $C$. difficile infection in hamsters. ${ }^{28}$ All the animals treated with oritavancin prior to $C$. difficile spore exposure survived for 20 days (duration of the trial), with no evidence of spore germination or toxin production in their ceca. In contrast, none of the hamsters pretreated with clindamycin or vancomycin survived beyond 6 days after $C$. difficile inoculation. Additionally, in an in vitro human gut model of $C$. difficile infection, oritavancin installation rapidly decreased the number of viable C. difficile and spore counts. ${ }^{28}$

In a mouse aerosol-anthrax model, a single-dose oritavancin was highly efficacious for both preexposure prophylaxis and postexposure treatment after challenge with Bacillus anthracis spores. ${ }^{29}$

\section{Clinical experience}

The clinical development of oritavancin has been delayed due to several transfers of drug ownership. Though the drug was originally developed by Eli Lily Research Laboratories in the mid1990s, the Phase III trials that led to the recent FDA approval of the drug were conducted by The Medicines Company. ${ }^{30}$

The two Phase III trials referred to as SOLO I and SOLO II compared the efficacy of single-dose IV oritavancin with IV vancomycin (twice daily for 7-10 days) for treatment of adults with ABSSSI. ${ }^{31,32}$ Both studies were designed as multicenter, randomized, double-blind trials and enrolled 1,959 patients. Enrolled patients were at least 18 years old with ABSSSI that included wound infection (either traumatic or surgical in origin), cellulitis, erysipelas, or a major skin abscess, suspected or proven to be due to a Gram-positive 
organism, that required treatment with at least 7 days of parenteral antibiotics. A surrounding erythema, edema, or an induration of at least $75 \mathrm{~cm}^{2}$ was required for the skin lesions to be eligible for both the studies. Patients were excluded for several reasons including necrotizing infections, severe sepsis, known or suspected bacteremia, animal bites, aquatic exposures, infected burns, diabetic foot infection, immunosuppression, and pregnancy. Patient characteristics including age, sex, race, type of infection, diabetes, hepatic impairment, and renal function were similar in both oritavancin and vancomycin groups. Patients were evaluated at 48-72 hours after starting drug (early clinical evaluation [ECE]), at the end of treatment (EOT), and at a post-therapy evaluation (7-14 days after EOT visit). The trials were designed to assess three efficacy end points for a non-inferiority margin of $10 \%$. The primary end point was assessed at ECE and was achieved if the investigators observed a reduction or halting of spread of the lesion size from baseline, patients remained afebrile, and additional rescue antibiotics were not required. Secondary end points evaluated were clinical cure at post-therapy evaluation and a $20 \%$ or more reduction in lesion size at ECE. ${ }^{31,32}$

In SOLO I trial, a total of 954 patients were included in the modified intention-to-treat (mITT) population and underwent randomization with 475 patients assigned to receive oritavancin and 479 patients assigned to the vancomycin arm. ${ }^{31}$ Approximately $83 \%$ of patients in both arms completed treatment and were included in the clinical evaluation (CE). The type of infections was well matched in both arms with approximately $50 \%$ of patients having cellulitis,
$30 \%$ having abscess, and 20\% having wound infection. Approximately $20 \%$ of patients in both oritavancin and vancomycin arms had diabetes mellitus, and about $34 \%$ of patients in both groups had a body mass index (BMI) of 30 or more. In approximately $60 \%$ of the patients in both treatment groups, a pathogen was isolated. Majority (96\%) of the identified pathogens were Gram-positive known to cause ABSSSI. Most common isolated pathogen was $S$. aureus, of which $48 \%$ was MRSA. Response to therapy for the mITT and CE population is summarized in Table 4. Similar efficacy was observed for primary and secondary end points in patients infected with MRSA in the intention-to-treat population with microbiologic evaluation. No significant difference with regard to the primary and secondary end points was noted both in the oritavancin and vancomycin arms for patients with a BMI of 30 or more compared with patients with a BMI of 30 or less. Likewise, patients with diabetes mellitus demonstrated similar response rate between the two treatment groups. ${ }^{31}$

In SOLO II trial, a total of 1,005 patients were included in the mITT population, of which 503 patients were randomized to the oritavancin arm, while 502 patients received IV vancomycin. ${ }^{32}$ Among the 835 patients who completed treatment, $85 \%(427 / 503)$ of patients were in the oritavancin arm, and $81 \%(408 / 502)$ belonged to the vancomycin arm. Approximately $9 \%$ of patients in both oritavancin and vancomycin arms were diabetic, and about $24 \%$ of patients in both groups had a BMI of 30 or more. Infection types in both the oritavancin and vancomycin arms were quite similar with approximately $30.9 \%$ cellulitis, $32.5 \%$ abscess, and $36.5 \%$ wound infection. The median baseline lesion size

Table 4 Oritavancin Phase III clinical trials

\begin{tabular}{|c|c|c|c|c|c|c|}
\hline & \multicolumn{3}{|l|}{ SOLO II } & \multicolumn{3}{|l|}{ SOLO II } \\
\hline & Oritavancin & Vancomycin & $\begin{array}{l}\text { Difference } \\
{[95 \% \mathrm{Cl}]}\end{array}$ & Oritavancin & Vancomycin & $\begin{array}{l}\text { Difference } \\
{[95 \% \mathrm{Cl}]}\end{array}$ \\
\hline \multicolumn{7}{|c|}{ Primary and secondary end points for mITT population } \\
\hline Primary composite end point & $82.3 \%(391 / 475)$ & $78.9 \%(378 / 479)$ & $3.4[-1.6,8.4]$ & $80.1 \%(403 / 503)$ & $82.9 \%(416 / 502)$ & $-2.7 \%[-7.5,2.0]$ \\
\hline$\geq 20 \%$ reduction in lesion size & $86.9 \%(4 \mid 3 / 475)$ & $82.9 \%(397 / 479)$ & $4.1[-0.5,8.6]$ & $85.9 \%(432 / 503)$ & $85.3 \%(428 / 502)$ & $0.6[-3.7,5.0]$ \\
\hline Clinical cure & $79.6 \%(378 / 475)$ & $80.0 \%(383 / 479)$ & $-0.4[-5.5,4.7]$ & $82.7 \%(416 / 503)$ & $80.5 \%(404 / 502)$ & $2.2[-2.6,7.0]$ \\
\hline \multicolumn{7}{|c|}{ Primary and secondary end points for CE population } \\
\hline Primary composite end point & $87.3 \%(344 / 394)$ & $86.1 \%(342 / 397)$ & $1.2[-3.6,5.9]$ & $83.6 \%(357 / 427)$ & $87.7 \%(358 / 408)$ & $-4.1[-8.9,6]$ \\
\hline$\geq 20 \%$ reduction in lesion size & $91.9 \%(362 / 394)$ & $93.2 \%(370 / 397)$ & $-1.3[-5.0,2.3]$ & $88.5 \%(378 / 427)$ & $89.2 \%(364 / 408)$ & $-0.7[-5.0,3.6]$ \\
\hline Clinical cure & $90.6 \%(357 / 394)$ & $88.7 \%(352 / 397)$ & $1.9[-2.3,6.2]$ & $93.2 \%(398 / 427)$ & $94.9 \%(387 / 408)$ & $-1.6[-4.9,1.6]$ \\
\hline \multicolumn{7}{|c|}{ Primary efficacy outcome at early clinical evaluation according to pathogen detected at baseline } \\
\hline Detection of at least one pathogen & $82.4 \%(201 / 244)$ & $81.0 \%(196 / 242)$ & I.4 $[-5.5,8.3]$ & $82.1 \%(234 / 285)$ & $85.1 \%(252 / 296)$ & $-3.0[-9.0,3.0]$ \\
\hline Staphylococcus aureus & $81.8 \%(180 / 220)$ & $81.9 \%(172 / 210)$ & $-0.1[-7.4,7.2]$ & $83.2 \%(208 / 250)$ & $84.9 \%(219 / 258)$ & $-1.7[-8.1,4.7]$ \\
\hline MRSA & $80.8 \%(84 / 104)$ & $80.0 \%(80 / 100)$ & $0.8[-10.1,11.7]$ & $82.0 \%(82 / 100)$ & $81.2 \%(82 / 101)$ & $0.8[-9.9,11.5]$ \\
\hline MSSA & $82.8 \%(96 / 116)$ & $83.6 \%(92 / 110)$ & $-0.9[-10.6,8.9]$ & $84.0 \%(126 / 150)$ & $87.3 \%(137 / 157)$ & $-3.3[-\mid I . I, 4.6]$ \\
\hline Enterococcus faecalis & $85.7 \%(6 / 7)$ & $80.0 \%(4 / 5)$ & NA & $83.3 \%(5 / 6)$ & $85.7 \%(6 / 7)$ & NA \\
\hline
\end{tabular}

Abbreviations: $\mathrm{Cl}$, confidence interval; mITT, modified intention to treat; CE, clinical evaluation; MRSA, methicillin-resistant Staphylococcus aureus; MSSA, methicillinsusceptible S. aureus; NA, not available. 
was $287.8 \mathrm{~cm}^{2}$ for the oritavancin group and $308.8 \mathrm{~cm}^{2}$ for the vancomycin group. A baseline pathogen was identified from approximately $70 \%$ of the treatment groups, and $97 \%$ of the isolated pathogens were Gram-positive organisms known to cause ABSSSIs. As expected, S. aureus was the most common pathogen. Response to therapy for the mITT and CE population is summarized in Table 4. There was no difference in efficacy outcomes between the oritavancin and vancomycin treatment groups in patients with a BMI of 30 or more. However, in the subgroup of patients with diabetes mellitus, a difference in response rate was observed between the two treatment arms. In patients with diabetes mellitus, the primary efficacy outcome at ECE was $73.9 \%$ (34/46) for the oritavancin arm versus $84.4 \%$ (38/45) for the vancomycin arm, and the secondary efficacy outcome at posttreatment evaluation was $69.6 \%(32 / 46)$ for the oritavancin arm versus $88.9 \%(40 / 45)$ for the vancomycin arm..$^{32}$ Considering the relatively small number of diabetic patients in the subgroup analysis, the authors highlight the need for some caution in interpreting the results, especially since the larger diabetic cohort in the SOLO I trial showed similar response rate between the two treatment groups.

In conclusion, both SOLO I and SOLO II trials demonstrated that a single dose of IV oritavancin was clinically and microbiologically non-inferior to twice-daily vancomycin administered 7-10 days for treatment of ABSSSIs cause by Gram-positive pathogens and the response rates were similar when analyzed by age, MRSA, sex, BMI, and race. ${ }^{31,32}$

\section{Safety profile}

In both SOLO I and SOLO II trials, safety profile was evaluated for up to 60 days considering the prolonged half-life of oritavancin. ${ }^{31,32}$ Study drug-related adverse event was encountered in $22.2 \%(217 / 976)$ of patients in the oritavancin arm versus $28.4 \%$ (279/983) of patients in the vancomycin arm. Adverse events leading to discontinuation of drug occurred in $3.7 \%$ (36/976) of patients in the oritavancin group compared to $4.2 \%(41 / 983)$ of patients in the vancomycin group. Serious adverse events (5.8\% and 5.9\% in the oritavancin and vancomycin arm, respectively) were almost identical. Most of the serious side effects were not related to the study medications.

The most common adverse effect encountered in either arm was nausea, with $9.9 \%(97 / 976)$ of patients in the oritavancin group and $10.5 \%(103 / 983)$ of patients in the vancomycin group overall reporting the symptom. Headache was the next most common adverse effect reported by $7.1 \%(69 / 976)$ of patients in the oritavancin arm and $6.7 \%(66 / 983)$ of patients in the vancomycin arm. Other observed adverse reactions were mild and fairly similar in both the oritavancin and vancomycin arms and are summarized in Table 5. Laboratory abnormalities were also similar, and no clinically relevant difference was noted in both treatment arms. Transient elevation of liver enzymes observed in both treatment groups did not result in discontinuation of either oritavancin or vancomycin.

\section{Formulary considerations}

The emergence of MRSA as the leading cause of complicated ABSSSIs in the USA and other countries has resulted in a significant shift in empiric antibiotic-prescribing patterns among clinicians..$^{33,34}$ This is particularly true for purulent wound infections and cellulitis, where a distinct shift in choice of empirical therapy from $\beta$-lactam antibiotics to those with activity against MRSA has occurred. ${ }^{35}$ Most of these infections do not require IV antibiotics and can usually be treated with oral antibiotics in the ambulatory setting. However, patients who do not fulfill criteria for hospital admission and fail or cannot tolerate oral therapy pose a unique challenge. These select groups of patients may be candidates for outpatient parenteral antimicrobial therapy (OPAT). ${ }^{36}$

The recent approval of oritavancin by the FDA has expanded the therapeutic armamentarium for clinicians for

Table 5 Adverse events attributed to oritavancin compared to vancomycin in Phase III trials

\begin{tabular}{|c|c|c|}
\hline \multirow{2}{*}{$\begin{array}{l}\text { Frequently reported } \\
\text { adverse events }\end{array}$} & \multirow{2}{*}{$\begin{array}{l}\text { Oritavancin } \\
\text { SOLO I + SOLO II } \\
(n=976)\end{array}$} & \multirow{2}{*}{$\begin{array}{l}\text { Vancomycin } \\
\text { SOLO I + SOLO II } \\
(n=983)\end{array}$} \\
\hline & & \\
\hline Nausea & $9.9 \%(97 / 976)$ & $10.5 \%(103 / 983)$ \\
\hline Headache & $7.1 \%(69 / 976)$ & $6.7 \%(66 / 983)$ \\
\hline Pruritus & $3 \%(29 / 976)$ & $7.4 \%(73 / 983)$ \\
\hline Vomiting & $4.6 \%(45 / 976)$ & $4.7 \%(46 / 983)$ \\
\hline Constipation & $3.4 \%(33 / 976)$ & $3.9 \%(38 / 983)$ \\
\hline Diarrhea & $3.7 \%(36 / 976)$ & $3.3 \%(32 / 983)$ \\
\hline Cellulitis & $3.8 \%(37 / 976)$ & $3.3 \%(32 / 983)$ \\
\hline Infusion-site extravasation & $3.4 \%(33 / 976)$ & $3.4 \%(33 / 983)$ \\
\hline Pyrexia & $3.1 \%(30 / 976)$ & $3.2 \%(31 / 983)$ \\
\hline Abscess on limb & $2.8 \%(27 / 976)$ & $1.3 \%(13 / 983)$ \\
\hline Dizziness & $2.7 \%(26 / 976)$ & $2.6 \%(26 / 983)$ \\
\hline Infusion-site phlebitis & $2.5 \%(24 / 976)$ & $1.5 \%(15 / 983)$ \\
\hline Infusion-site reaction & $1.9 \%(19 / 976)$ & $3.5 \%(34 / 983)$ \\
\hline Insomnia & $1.4 \%(14 / 976)$ & $1.3 \%(13 / 983)$ \\
\hline Chills & $1 \%(10 / 976)$ & $1.2 \%(12 / 983)$ \\
\hline Fatigue & $1 \%(10 / 976)$ & $0.6 \%(6 / 983)$ \\
\hline Urticaria & $0.7 \%(7 / 976)$ & $1.5 \%(15 / 983)$ \\
\hline $\begin{array}{l}\text { Alanine aminotransferase } \\
\text { elevation }\end{array}$ & $2.8 \%(27 / 976)$ & $1.5 \%(15 / 983)$ \\
\hline $\begin{array}{l}\text { Aspartate } \\
\text { aminotransferase elevation }\end{array}$ & I.1\% (I I/976) & I.I\% (I I/983) \\
\hline
\end{tabular}


treatment of ABSSSIs. A single IV injection over 3 hours, without the need for a peripherally inserted central catheter, makes oritavancin an ideal candidate for use in an ambulatory clinic or urgent care setting. There are no significant interactions with other drugs, and dose adjustments for renal and hepatic impairments are not required. Unlike vancomycin, drug-level monitoring is not required after administration of oritavancin. Overall, this can improve quality of life and patient satisfaction and enable patients to return to work faster. ${ }^{37}$ The long half-life of the drug has the potential to reduce relapse rates, and the multifaceted mechanism of action will possibly prevent and likely delay development of resistance. Finally, a single-dose regimen eliminates problems associated with patient compliance which may be encountered with daily or even multiple intermittent doses.

However, the benefits of a single dose do pose some unique challenges with oritavancin that clinicians need to consider. The long half-life of the drug does put patients at risk of prolonged injury if a delayed hypersensitivity reaction or other adverse reactions occur. Fortunately, side effects due to oritavancin in clinical trials were mostly mild, and serious adverse events attributable to the drug were extremely rare. One potential disadvantage of a single-dose drug administered in an ambulatory setting might be the lack of rigorous clinical monitoring that is required in treating a complicated wound infection. This model thus relies on patients to care for them and makes patients and their caregivers more responsible to seek help if the clinical situation worsens. Though this strategy may work for majority of patients, the authors do anticipate some problems in certain subgroups (elderly patients, morbidly obese patients, patients with vascular insufficiency). Additionally, complicated wound

Table 6 Some intravenous antibiotics for acute bacterial skin and skin structure infections in adults

\begin{tabular}{|c|c|c|}
\hline Drug & Usual adult dose & $\begin{array}{l}\text { Cost of 7-day } \\
\text { therapy }^{\mathrm{a}}\end{array}$ \\
\hline Vancomycin & $\begin{array}{l}\text { I5-20 mg/kg (max } 2 \text { g) q } 12 \text { hours } \\
\times 7-14 \text { days }^{\text {b }}\end{array}$ & $\$ 115.50$ \\
\hline Dalbavancin & $\mathrm{I}, 000 \mathrm{mg} \times \mathrm{I}$, then $500 \mathrm{mg} \mathrm{I}$ week later & $\$ 2,980.00^{c}$ \\
\hline Oritavancin & $\mathrm{I}, 200 \mathrm{mg}$ once & $\$ 2,900.00^{d}$ \\
\hline Televancin & 10 mg/kg q 24 hours $\times 7-14$ days $^{\mathrm{b}}$ & $\$ 2,166.50$ \\
\hline Linezolid & $600 \mathrm{mg} q 12$ hours $\times 10-14$ days & $\$ 1,952.30$ \\
\hline Tedizolid & $200 \mathrm{mg}$ q 24 hours $\times 6$ days & $\$ 1,410.00$ \\
\hline Ceftaroline & $600 \mathrm{mg} q 12$ hours $\times 5-14$ days $^{\mathrm{b}}$ & $\$ 1,768.90$ \\
\hline Daptomycin & $4 \mathrm{mg} / \mathrm{kg}$ q 24 hours $\times 7-14$ days $^{\mathrm{b}}$ & $\$ 2,482.90^{\mathrm{e}}$ \\
\hline
\end{tabular}

Notes: aAproximate wholesale acquisition cost for 7-day treatment of a $70 \mathrm{~kg}$ patient with the lowest usual dosage; bdosage adjustment may be needed for renal or hepatic impairment; 'cost for two $500 \mathrm{mg}$ vials; 'cost for three $400 \mathrm{mg}$ vials; ${ }^{\mathrm{e}}$ cost for one $500 \mathrm{mg}$ vial. Data from The Medical Letter, Inc.. ${ }^{2}$ infections can progress to bacteremia and bone and joint infections, where the efficacy of oritavancin has not been fully assessed.

The use of oritavancin in ABSSSIs is sure to reignite the debate over cost of therapy. Table 6 highlights the cost of therapy for the traditionally used and recently approved IV antibiotics in ABSSSIs. A single-dose treatment in an ambulatory setting does have the advantage of reducing the cost of OPAT and ongoing home care needs. However, since the offending organisms for majority of ABSSSIs are Gram-positive pathogens other than MRSA, one could argue about using a $\beta$-lactam antibiotic after a short empiric course of a cheaper IV antibiotic and thus reduce cost. Thus, there is a need for carefully conducted cost-benefit analysis of oritavancin compared to other antibiotics used for OPAT in ABSSSIs. Furthermore, additional clinical experience with other serious infections that warrant a longer course of therapy is needed before oritavancin use in the outpatient arena becomes widely accepted.

\section{Conclusion}

Outpatient therapy with single-dose oritavancin offers a new paradigm in treatment of ABSSSI with the potential to reduce cost of care and improve patient satisfaction. The drug has a similar safety profile as vancomycin with comparable cure rates for complicated skin infections. The high acquisition cost of the drug has to be considered in conjunction with several potential advantages over other drugs. Oritavancin would likely be a useful armamentarium in the physician toolkit for treatment of complicated ABSSSI caused by Gram-positive pathogens including MRSA once the reimbursement and co-pay issues have been resolved.

\section{Disclosure}

The authors report no conflicts of interest in this work.

\section{References}

1. Edelsberg J, Weycker D, Barron R, et al. Prevalence of antibiotic resistance in US hospitals. Diagn Microbiol Infect Dis. 2014;78:255-262.

2. Oritavancin (orbactiv) for skin and skin structure infections. Med Lett Drugs Ther. 2015;57:3-5.

3. ORBACTIV Tм (Oritavancin) For Injection. Parsippany, NJ: The Medicines Company; 2014. [Prescribing Information].

4. Zhanel GG, Schweizer F, Karlowsky JA. Oritavancin: mechanism of action. Clin Infect Dis. 2012;54(Suppl 3):S214-S219.

5. Groves P, Searle MS, Mackay JP, Williams DH. The structure of an asymmetric dimer relevant to the mode of action of the glycopeptide antibiotics. Structure. 1994;2:747-754.

6. Allen NE, Nicas TI. Mechanism of action of oritavancin and related glycopeptide antibiotics. FEMS Microbiol Rev. 2003;26:511-532. 
7. Belley A, Neesham-Grenon E, McKay G, et al. Oritavancin kills stationary-phase and biofilm Staphylococcus aureus cells in vitro. Antimicrob Agents Chemother. 2009;53:918-925.

8. Belley A, Neesham-Grenon E, Arhin FF, McKay GA, Parr TR Jr, Moeck G. Assessment by time-kill methodology of the synergistic effects of oritavancin in combination with other antimicrobial agents against Staphylococcus aureus. Antimicrob Agents Chemother. 2008;52: 3820-3822.

9. Belley A, McKay GA, Arhin FF, et al. Oritavancin disrupts membrane integrity of Staphylococcus aureus and vancomycin-resistant enterococci to effect rapid bacterial killing. Antimicrob Agents Chemother. 2010;54:5369-5371.

10. Baquir B, Lemaire S, Van Bambeke F, Tulkens PM, Lin L, Spellberg B. Macrophage killing of bacterial and fungal pathogens is not inhibited by intense intracellular accumulation of the lipoglycopeptide antibiotic oritavancin. Clin Infect Dis. 2012;54(Suppl 3):S229-S232.

11. Citron DM, Kwok YY, Appleman MD. In vitro activity of oritavancin (ly333328), vancomycin, clindamycin, and metronidazole against Clostridium perfringens, Propionibacterium acnes, and anaerobic gram-positive cocci. Anaerobe. 2005;11:93-95.

12. Mendes RE, Farrell DJ, Sader HS, Flamm RK, Jones RN. Activity of oritavancin against gram-positive clinical isolates responsible for documented skin and soft-tissue infections in European and US hospitals (2010-2013). J Antimicrob Chemother. 2015;70:498-504.

13. Karlowsky JA, Walkty AJ, Baxter MR, Adam HJ, Zhanel GG. In vitro activity of oritavancin against gram-positive pathogens isolated in Canadian hospital laboratories from 2011 to 2013. Diagn Microbiol Infect Dis. 2014;80:311-315.

14. Arhin FF, Sarmiento I, Belley A, et al. Effect of polysorbate 80 on oritavancin binding to plastic surfaces: implications for susceptibility testing. Antimicrob Agents Chemother. 2008;52:1597-1603.

15. Patti GJ, Kim SJ, Yu TY, et al. Vancomycin and oritavancin have different modes of action in enterococcus faecium. J Mol Biol. 2009;392: 1178-1191.

16. Arthur M, Depardieu F, Reynolds P, Courvalin P. Moderate-level resistance to glycopeptide ly333328 mediated by genes of the VanA and $\operatorname{VanB}$ clusters in enterococci. Antimicrob Agents Chemother. 1999;43: 1875-1880.

17. Rubino CM, Van Wart SA, Bhavnani SM, Ambrose PG, McCollam JS, Forrest A. Oritavancin population pharmacokinetics in healthy subjects and patients with complicated skin and skin structure infections or bacteremia. Antimicrob Agents Chemother. 2009;53: 4422-4428.

18. Ambrose PG, Drusano GL, Craig WA. In vivo activity of oritavancin in animal infection models and rationale for a new dosing regimen in humans. Clin Infect Dis. 2012;54(Supp1 3):S220-S228.

19. Bhavnani SM, Owen JS, Loutit JS, Porter SB, Ambrose PG. Pharmacokinetics, safety, and tolerability of ascending single intravenous doses of oritavancin administered to healthy human subjects. Diagn Microbiol Infect Dis. 2004;50:95-102.

20. Fetterly GJ, Ong CM, Bhavnani SM, et al. Pharmacokinetics of oritavancin in plasma and skin blister fluid following administration of a 200-milligram dose for 3 days or a single 800-milligram dose. Antimicrob Agents Chemother. 2005;49:148-152.

21. Boylan CJ, Campanale K, Iversen PW, Phillips DL, Zeckel ML, ParrTR Jr. Pharmacodynamics of oritavancin (ly333328) in a neutropenic-mouse thigh model of Staphylococcus aureus infection. Antimicrob Agents Chemother. 2003;47:1700-1706.

Infection and Drug Resistance

\section{Publish your work in this journal}

Infection and Drug Resistance is an international, peer-reviewed openaccess journal that focuses on the optimal treatment of infection (bacterial, fungal and viral) and the development and institution of preventive strategies to minimize the development and spread of resistance. The journal is specifically concerned with the epidemiology of antibiotic
22. Rupp ME, Fey PD, Longo GM. Effect of ly 333328 against vancomycin-resistant Enterococcus faecium in a rat central venous catheter-associated infection model. J Antimicrob Chemother. 2001;47:705-707.

23. Kaatz GW, Seo SM, Aeschlimann JR, Houlihan HH, Mercier RC, Rybak MJ. Efficacy of ly333328 against experimental methicillinresistant Staphylococcus aureus endocarditis. Antimicrob Agents Chemother. 1998;42:981-983.

24. Saleh-Mghir A, Lefort A, Petegnief Y, et al. Activity and diffusion of ly333328 in experimental endocarditis due to vancomycin-resistant Enterococcus faecalis. Antimicrob Agents Chemother. 1999;43: $115-120$.

25. Lefort A, Saleh-Mghir A, Garry L, Carbon C, Fantin B. Activity of ly333328 combined with gentamicin in vitro and in rabbit experimental endocarditis due to vancomycin-susceptible or -resistant Enterococcus faecalis. Antimicrob Agents Chemother. 2000;44:3017-3021.

26. Gerber J, Smirnov A, Wellmer A, et al. Activity of ly333328 in experimental meningitis caused by a Streptococcus pneumoniae strain susceptible to penicillin. Antimicrob Agents Chemother. 2001;45: 2169-2172.

27. Cabellos C, Fernàndez A, Maiques JM, et al. Experimental study of ly333328 (oritavancin), alone and in combination, in therapy of cephalosporin-resistant pneumococcal meningitis. Antimicrob Agents Chemother. 2003;47:1907-1911.

28. Freeman J, Marquis M, Crowther GS, et al. Oritavancin does not induce Clostridium difficile germination and toxin production in hamsters or a human gut model. J Antimicrob Chemother. 2012;67:2919-2926.

29. Heine HS, Bassett J, Miller L, et al. Efficacy of oritavancin in a murine model of bacillus anthracis spore inhalation anthrax. Antimicrob Agents Chemother. 2008;52:3350-3357.

30. Allen NE. From vancomycin to oritavancin: the discovery and development of a novel lipoglycopeptide antibiotic. Anti Infect Agents Med Chem. 2010;9:23-47.

31. Corey GR, Kabler H, Mehra P, et al; SOLO I Investigators. Single-dose oritavancin in the treatment of acute bacterial skin infections. $N$ Engl J Med. 2014;370:2180-2190.

32. Corey GR, Good S, Jiang H, et al; SOLO II Investigators. Single-dose oritavancin versus 7-10 days of vancomycin in the treatment of grampositive acute bacterial skin and skin structure infections: the SOLO II noninferiority study. Clin Infect Dis. 2015;60:254-262.

33. Talan DA, Krishnadasan A, Gorwitz RJ, et al; EMERGEncy ID Net Study Group. Comparison of Staphylococcus aureus from skin and soft-tissue infections in us emergency department patients, 2004 and 2008. Clin Infect Dis. 2011;53:144-149.

34. Moran GJ, Krishnadasan A, Gorwitz RJ, et al; EMERGEncy ID Net Study Group. Methicillin-resistant $S$. aureus infections among patients in the emergency department. N Engl J Med. 2006;355:666-674.

35. Singer AJ, Talan DA. Management of skin abscesses in the era of methicillin-resistant Staphylococcus aureus. N Engl J Med. 2014;370: 1039-1047.

36. Tice AD, Rehm SJ. Meeting the challenges of methicillin-resistant Staphylococcus aureus with outpatient parenteral antimicrobial therapy. Clin Infect Dis. 2010;51(Supp1 2):S171-S175.

37. Tice A. Oritavancin: a new opportunity for outpatient therapy of serious infections. Clin Infect Dis. 2012;54(Suppl 3):S239-S243.

resistance and the mechanisms of resistance development and diffusion in both hospitals and the community. The manuscript management system is completely online and includes a very quick and fair peerreview system, which is all easy to use. Visit http://www.dovepress.com/ testimonials.php to read real quotes from published authors. 